\title{
OBSERVATIONS OF DIFFUSE INFRARED RADIATION BY A SMALL CRYOGENICAL TELESCOPE, IRTS
}

\author{
Haruyuki Okuda \\ Institute of Space and Astronautical Science \\ 3-1-1 Yoshinodai Sagamihara \\ Kanagawa \#229, Japan
}

\begin{abstract}
A small, cryogenically cooled infrared telescope named IRTS (Infrared Telescope in Space) is under development. The telescope is equipped with spectrometers and a photometer with medium spatial and spectral resolution covering the wavelength range from 1 to $1000 \mu \mathrm{m}$. The instrument is specially designed for observations of the cosmic background radiation and galactic infrared radiation. It will be flown on board a Japanese reusable free-flyer called SFU (space flyer unit) to be launched in 1994.
\end{abstract}

\section{INTRODUCTION}

Infrared observations from space are free from atmospheric absorption and emission. In particular, the absence of atmospheric emission enables us to utilize fully the capabilities of the infrared detectors. These advantages have been demonstrated by the success of the IRAS satellite with its many discoveries.

Stimulated by the success of IRAS, several follow-on missions are under preparation or in planning. COBE is an infrared explorer dedicated to observations of the cosmic background radiation. It is equipped with a far-infrared and submillimeter spectrometer with a mediumsize beam and a multiband photometer with a narrow beam. It will be launched in the summer of 1989 and used for photometric mapping and spectroscopic measurements of the cosmic background radiation. ISO is an observatory-type facility equipped with various types of instruments, including an imaging camera, multiband photometers, and medium and high resolution spectrometers. It is scheduled to be launched in 1993. A similar facility called SIRTF is also planned by NASA. These two facilities will be used primarily for detailed observations of individual sources.

As a complementary mission to the above investigations, we are preparing a small, cryogenically cooled telescope for observations of diffuse infrared radiation.

\section{THE IRTS PROJECT}

IRTS is a small, cryogenically cooled telescope with a medium spectral and spatial resolution, which will be used mainly for observations of diffuse components of galactic and extragalactic infrared radiation. A general view of IRTS is shown in Figure 1. The telescope employs F/4 Ritchy-Chretian optics with a $15 \mathrm{~cm}$ primary. Four kinds of instruments are installed at the focal point, and the beam is directed to these with pyramidal mirrors. They can be operated simultaneously, in adjacent positions. For rejection of stray radiation, we use two types of baffling in series: a combination of a paraboloidal forebaffle with a specular surface and a conventional aftbarrel with blackened wall and fins. The specularly reflective baffle is especially advantageous, since it is hardly warmed when illuminated with external radiation, and even when warmed, its thermal emission is low. 


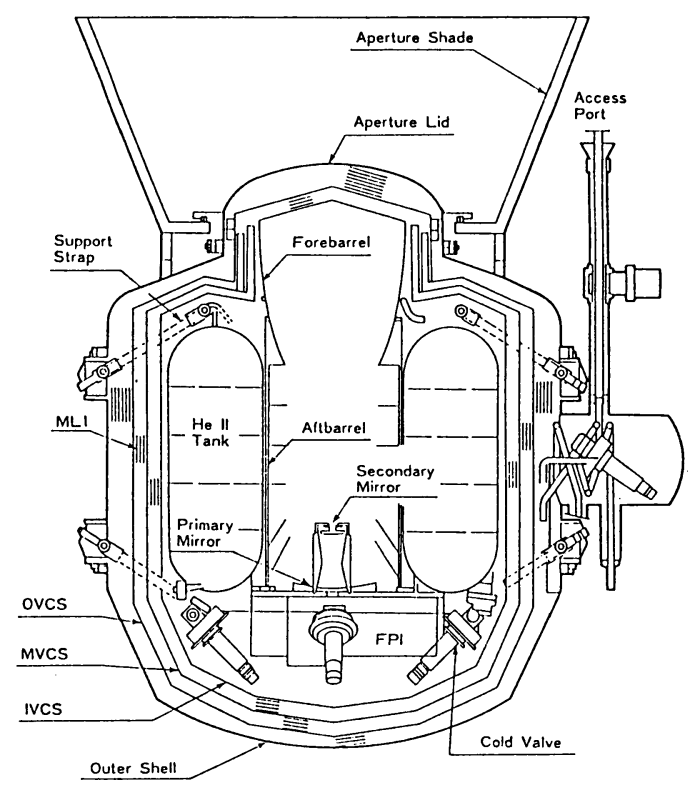

Figure 1. The IRTS instrument.

The total system is enshrouded in a liquid helium tank and cooled to superfluid helium temperature. The capacity of the helium tank is 100 liters, which is sufficient for about one month's observing. Evaporated helium gas is vented through a porous plug made with sintered ceramics. The liquid helium tank, together with the telescope, the focal plane instruments, and the multilayered radiation shield, is suspended by glass fiber reinforced plastic straps from the vacuum container.

The characteristics of the four instruments are summarized in Table 1. NIRS is a grating spectrometer using a multi-element InSb array for medium resolution spectroscopy in the nearinfrared. MIRS is a single concave grating spectrometer combined with a SiGa detector array that provides medium-resolution spectroscopy in the middle-infrared region. FILM is a narrow-band grating monochromator tuned for the CII line at $158 \mu \mathrm{m}$ and the OI line at $63 \mu \mathrm{m}$. This will be used for mapping of line intensities in the galactic plane. FIRP is a multiband filter photometer in the far-infrared and submillimeter region. ${ }^{3} \mathrm{He}$ cooled bolometers are used for the detectors. All the instruments have a medium beam size of $0.1-0.2^{\circ}$.

The telescope is installed in a side-looking position, and observations are performed by scanning along a great circle while rotating the SFU with a constant speed synchronized with its orbital revolution. The rotation axis is always kept pointing to the sun, and thus the scanning plane sweeps the sky secularly with the motion of the sun. During the observation time of about two weeks allotted for IRTS, about $10 \%$ of the sky will be covered.

\section{SCIENTIFIC OBJECTIVES}

Observations by IRTS will be devoted to spectroscopic and photometric measurements of diffuse infrared emissions of the sky. These are a natural extension of the balloon and rocket observations in which we have been engaged in the past decades. Major scientific objectives 
TABLE 1. Focal Plane Instruments of IRTS

\begin{tabular}{|c|c|c|c|c|}
\hline Instrument & NIRS & MIRS & $F I L M$ & FIRP \\
\hline Optical & Grating & Grating & Grating & Multiband \\
\hline System & Spectrometer & Spectrometer & Spectrometer & Photometer \\
\hline $\begin{array}{l}\text { Wavelength } \\
\text { Range }(\mu \mathrm{m})\end{array}$ & $1.2-4.1$ & $5-13.5$ & $\begin{array}{l}63.2(\mathrm{O} I) \\
157.7(\mathrm{C} \mathrm{II})\end{array}$ & $60-1000$ \\
\hline $\begin{array}{l}\text { Spectral } \\
\text { Resolution }\end{array}$ & $0.15 \mu \mathrm{m}$ & $0.26 \mu \mathrm{m}$ & $\lambda \Delta \lambda=400$ & $\lambda / \Delta \lambda=0.4$ \\
\hline Beam Size & $0.14^{\circ} \times 0.14^{\circ}$ & $0.14^{\circ} \times 0.14^{\circ}$ & $0.14^{\circ} \times 0.3^{\circ}$ & $0.5^{\circ}$ \\
\hline Detector & $\begin{array}{l}\text { InSb Arrays } \\
24 \mathrm{el} .\end{array}$ & $\begin{array}{l}\text { SiGa Arrays } \\
32 \text { el. }\end{array}$ & $\begin{array}{l}\text { Ge:Ga } \times 4 \\
\text { (Stressed 3) }\end{array}$ & $\begin{array}{l}{ }^{3} \mathrm{He} \text { Bolometer } \\
\times 6\end{array}$ \\
\hline Pre-Amp & DRO/CIA* & DRO/CIA & TIA & TIA \\
\hline $\begin{array}{l}\text { Detection Limit } \\
\mathrm{W} \mathrm{cm}^{2} \mu \mathrm{m}^{-1} \mathrm{sr}^{-1}\end{array}$ & $\begin{array}{l}2.8 \times 10^{-13} \\
(1 \mathrm{sec}, 1 \sigma)\end{array}$ & $\begin{array}{l}3 \times 10^{-14} \\
(1 \mathrm{sec}, 1 \sigma)\end{array}$ & $\begin{array}{l}5 \times 10^{-14} \\
(1 \mathrm{sec}, 1 \sigma)\end{array}$ & $\begin{array}{l}5 \times 10^{-14} \\
(1 \mathrm{sec}, 1 \sigma)\end{array}$ \\
\hline $\begin{array}{l}\text { Main } \\
\text { Objectives }\end{array}$ & $\begin{array}{l}\text { Cosmic background, } \\
\text { Galaxy, dust }\end{array}$ & $\begin{array}{l}\text { Gases, dust, } \\
\text { zodiacal light }\end{array}$ & $\begin{array}{l}\text { Interstellar } \\
\text { gases }\end{array}$ & $\begin{array}{l}\text { Cosmic background, } \\
\text { IR-Cirrus }\end{array}$ \\
\hline
\end{tabular}

*Direct read-out/charge integration amplifier, †Trans impedance amplifier

of the observations are outlined in the following.

\subsection{Cosmic Background Radiation}

One of the major targets of the observations will be measurements of the cosmic background radiation. In the history of the universe, there is a big gap in our knowledge between the age of the $3 \mathrm{~K}$ background radiation $(z \sim 1000)$ and that of quasars $(z \sim 4)$. In these missing ages, the first-generation stars and galaxies should have been born. The light emitted in this period, supposed to be mostly emitted in the ultraviolet (UV) and visible regions, would be observed in the near-infrared as a result of the large redshift. In this respect, observations of near-infrared background radiation would bring crucial information to the study of the early history of the universe. The group at Nagoya University has carried out an experiment in search of near-infrared background radiation (Matsumoto, Akiba, and Murakami, 1988). The observed spectrum in the rocket experiment (after careful subtraction of foreground emissions such as the zodiacal light, stellar light, and airglow emission) shows appreciably higher intensities than expected from the conventional theory of the early universe. The rocket experiments, however, provided only a short observation time and might be contaminated with the emission of exhaust gases from the rocket engine. More observations are clearly needed.

Remarkable improvements in observational conditions will be expected with IRTS. The larger telescope aperture of $15 \mathrm{~cm}$, with a finer spatial resolution of $0.1^{\circ}$, will allow better discrimination of the contribution of stellar light. The longer observation time will allow selection of data to attain minimum effects of stellar light and the zodiacal light.

The finer spectral resolution available in NIRS will be useful not only for the finer determination of continuum spectra, but it will also enhance the possibility of detecting a sharp enhancement near $2 \mu \mathrm{m}$ that has been tentatively found in the observed spectrum of Matsumoto et al. If such a line feature is present, it could be used for identification of the time and period of the relevant activities in the early history of the universe. 
Observations of the $3 \mathrm{~K}$ background radiation are also very important for exploring the early history of the universe. In particular, the Wien portion of the spectrum may have been modulated in later stages of evolution. The excess observed by Matsumoto et al. (1988) may be due to an inverse Compton effect, which would have enhanced the energy spectrum in shorter wavelengths. Alternately, thermal emission of dust particles produced in firstgeneration star formation may have added an extra component in the submillimeter range (Hayakawa et al., 1987). Either mechanism should introduce some anisotropy in the intensity distribution, together with the modification of the energy spectrum.

\subsection{Galactic Structure and Interstellar Physics}

The Galaxy is a grand composite of stars, gases, and dust. Integrated sky light is therefore the sum of the contributions of all these components, which obviously depends on wavelength.

In the near-infrared, the emission is dominated by stellar light, and thus its observation is useful in delineating the distribution of stars in the Galaxy. In fact, the near-infrared surveys of the galactic plane by balloons have clearly revealed the stellar distribution in the inner region of the Galaxy (e.g., Okuda, 1981), which had been obscured by strong interstellar extinction. The observations were, however, limited, and they explored only a single band of $2.4 \mu \mathrm{m}$, a unique band gap of the strong $\mathrm{OH}$ airglow emission. Wider coverage of wavelengths and spectroscopic information are necessary not only to distinguish the contribution of different types of stars, but also to correct interstellar extinction effects.

The near-infrared spectrometer has sufficient bandwidth $(1-5 \mu \mathrm{m}$, and resolution $(0.1 \mu \mathrm{m})$ to provide detailed observations of the spectral shape of stellar light and also to be useful for the detection of emission or absorption lines. For example, the detection of $\mathrm{CO}$ bands $(2.3 \mu \mathrm{m}, 4.6 \mu \mathrm{m})$ and $\mathrm{H}_{2} 0$-absorption $(2.7 \mu \mathrm{m})$ will help to estimate the contribution of dwarf and giant stars. The contribution of dwarf stars is important to estimate the total mass of the Galaxy.

In the far-infrared region, a main objective is the mapping of the galactic plane with [CII] $(158 \mu \mathrm{m})$ and [OI] $(63 \mu \mathrm{m})$ fine structure lines. Carbon is cosmically abundant and has a lower ionization potential $(11.3 \mathrm{eV})$ than hydrogen $(13.6 \mathrm{eV})$. Thus, the ionized carbon region (CII region) may be distributed more widely in interstellar space. In addition, the excitation temperature of the [CII] line is very low $(92 \mathrm{~K})$, and hence the emission would be found in regions with relatively low temperatures, but not so cold as that found in molecular clouds, such as at the boundaries between the HII region and the molecular cloud. The [CII] line plays an important role in the cooling of interstellar gases, and its emission would be very strong and extensively distributed in interstellar space. In fact, recent balloon observations have shown that this emission is present ubiquitously in the galactic plane (Okuda et al., 1989, this volume). The [CII] line would become a useful probe to study moderate temperature and moderate density gases, a newly discovered state of the interstellar medium. Survey work similar to that carried on at the $21 \mathrm{~cm}$ line (HI region) and at the $2.6 \mathrm{~mm} \mathrm{CO}$ line $\left(\mathrm{H}_{2}\right.$ region) may well be productive.

The [OI] line is also expected to be strongly emitted in interstellar space, but with somewhat higher temperatures and densities. The intensity ratio of [CII] and [OI] is therefore a good parameter for the determination of the temperature and density of interstellar gases. It is also suggested that $[\mathrm{OI}]$ emission is a good tracer of interstellar shock induced by expanding shells of supernova remnants or collisions of interstellar clouds. The FILM is designed so as to make simultaneous observations of both lines by using a self-contained beam splitter. 


\subsection{Cosmic Dust}

The Air Force Geophysical Laboratory rocket observations and IRAS observations have found that the galactic plane is much brighter in the middle-infrared region than expected from thermal emission of conventional interstellar dust. Similar emission was also observed in the near-infrared region in reflection nebulae and HII regions (Sellgren et al., 1985).

This emission is characterized by a high color temperature $(1000 \mathrm{~K})$ and is uniformly distributed throughout the emission region. This behavior has been interpreted by introducing extremely small grains of radius on the order of $10 \AA$, which are heated to a high transient temperature when a single UV photon is absorbed. The same dust particles are presumed to be responsible for the unidentified infrared bands $(3.3,3.4,6.2,7.7,8.6,11.3 \mu \mathrm{m}$, etc.) which have been found in the circumferences of planetary nebulae. Some exotic materials such as polycyclic aromatic hydrocarbon (Puget, Leger, and Boulanger, 1985) and quenched carbonaceous composite (Sakata et al., 1984) have been proposed for these particles. Observations with the MIRS, in combination with the NIRS, would provide valuable information on the nature of the emission and the emitting materials.

The same technique can be applied for observations of interplanetary dust emissions. The study of the interplanetary dust is very important to clarify the origin and evolution of the solar system.

In addition to observations of diffuse omission, IRTS is also useful for studies of discrete sources. Although the telescope is small, the detection limit is sufficiently low that thousands of stars will be incidentally observed in the course of the general survey of the sky. This unbiased photometric and spectroscopic sample will be valuable for the study of characteristics of stellar systems. In particular, these data will be useful for understanding the generation and evolution of circumstellar dust from a statistical study of a large number of stars in different stages of evolution.

In conclusion, IRTS is a small but valuable mission for infrared astronomy in the mid90s. It is designed for observations of the diffuse component of galactic and extragalactic infrared emissions. Its function is complementary to that of the COBE and ISO missions.

This mission is under development as a collaboration between Japan and the USA. Part of the instrumentation is being developed by US groups, and data acquisition is supported by the Deep Space Network at NASA.

\section{REFERENCES}

Hayakawa, S., Matsumoto, T., Matsuo, H., Murakami, H., Richards, P. L., and Sato, S. 1987, Publ. Astr. Soc. Japan, 39, 941.

Matsumoto, T., Akiba, M., and Murakami, H. 1988, Ap. J., 322, 575.

Matsumoto, T., Hayakawa, S., Matsuo, H., Murakami, H., Sato, S., Lange, A. E., and Richards, P. L. 1988, Ap. J., 329, 567.

Okuda, H. 1981, in IAU Symposium 96, Infrared Astronomy, ed. D. P. Cruicshank, and G. WynnWilliams, 247.

Okuda, H., et al. 1989, in IAU Symposium 139, Galactic and Extragalactic Background Radiation, ed. S. Bowyer and Ch. Leinert, Kluwer Academic Publishers, Dordrecht.

Puget, J. L., Leger, A., and Boulanger, F. 1985, Astro. Ap., 142, L19.

Sakata, A., Wada, S., Tanabe, T., and Onaka, T. 1984, Ap. J. Letters, 287, L51.

Sellgren, K., Allamandola, L. J., Bregman, J.. D., Werner, M. W., and Wooden, D. H. 1985, Ap. J., $299,416$. 